\title{
A single-arm feasibility cohort study of rivaroxaban in antiphospholipid syndrome
}

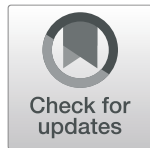

\author{
Kimberly Legault ${ }^{*}$, Mark Blostein², Marc Carrier ${ }^{3}$, Susan Kahn², Sam Schulman', Sudeep Shivakumar ${ }^{4}$, \\ Cynthia Wu ${ }^{5}$ and Mark A. Crowther ${ }^{1}$
}

\begin{abstract}
Background: There is uncertainty regarding the safety and effectiveness of direct oral anticoagulant agents in patients with antiphospholipid syndrome (APS). We performed a multicenter feasibility study to examine our ability to identify and obtain consent from eligible APS patients and to obtain $95 \%$ adherence with daily rivaroxaban administration, in order to inform and power a larger study. Clinical outcomes of bleeding and thrombosis were also collected.
\end{abstract}

Methods: APS patients with prior venous thromboembolism (VTE) were recruited over 2 years (Oct 2014-Sept 2016) and followed for $\geq 1$ year. Patients were assessed clinically every 3 months and had pill counts performed every 6 months. Numbers of patients fulfilling study criteria, as well as those consenting to participate, were tracked, and percentage adherence based on pill counts was recorded. These data were compared against the feasibility endpoints. Rates of thrombosis and bleeding were calculated. Criterion for feasibility was obtaining consent from 135 of 150 identified APS patients over 2 years.

Results: Ninety-six eligible patients were identified, and 14 declined participation. Eighty-two patients were followed for a mean of 19 months, representing 129.8 patient-years. Average rivaroxaban adherence was 95.0\%. During follow-up, there were 4 thromboembolic events (2 cerebrovascular and 2 VTE). There were no episodes of major bleeding.

Conclusions: Adequately powered comparative trials using patient-important outcomes in APS are unlikely to be successful due to inability to recruit sufficient numbers of study subjects. This study does not reveal a higher than expected risk of recurrent thromboembolic disease compared to historical cohorts; however, this is an uncontrolled study in relatively low-risk APS patients.

Trial registration: The study was registered with clinicaltrials.gov, identifier NCT02116036, April 16, 2014.

Keywords: Antiphospholipid syndrome, Feasibility studies, Hemorrhage, Rivaroxaban, Thromboembolism

\section{Introduction}

The antiphospholipid syndrome (APS) is characterized by clinical manifestations (venous or arterial thrombosis and/or recurrent pregnancy loss) occurring in patients with persistent antiphospholipid antibody positivity

\footnotetext{
* Correspondence: legauk2@mcmaster.ca

'Department of Medicine, McMaster University, 1280 Main St. W, Hamilton, ON L8S 4L8, Canada

Full list of author information is available at the end of the article
}

[either anticardiolipin antibody (aCL), lupus anticoagulant (LA, otherwise known as non-specific inhibitor), and/or anti-beta-2 glycoprotein-1 antibody (aß2GP1), positive on $\geq 2$ occasions $\geq 12$ weeks apart] [1]. APS is a common cause of myocardial infarction and stroke in patients under the age of 50 and is particularly prevalent in patients with autoimmune conditions, especially systemic lupus erythematosus (SLE) [2]. The prothrombotic state associated with APS may cause either arterial or 
venous thrombosis, with about $55 \%$ of reported events being either arterial or mixed arterial and venous [3]. Our multicenter collaborative research group has confirmed that the optimal therapy for the prevention of recurrent venous or arterial thrombosis in patients with APS is warfarin administered to achieve an international normalized ratio (INR) of 2.0-3.0 [4].

Although highly effective, warfarin requires frequent, careful monitoring [5]. Conversely, direct oral anticoagulants (DOACs) are replacing warfarin for many indications in Canada due to comparable efficacy for indications including prevention and treatment of venous thromboembolism (VTE) and stroke prevention in patients with nonvalvular atrial fibrillation, with comparative ease of use [6-9].

However, in patients with APS, simple extrapolation from other clinical situations (where these agents are safely substituted for warfarin) is not warranted given the unique characteristics of this population. First, some patients with APS seem to be at very high risk for recurrent thrombosis, suffering a higher than expected rate of recurrent events despite "usual intensity" therapy with warfarin $[10,11]$. Second, many patients with APS receive medications such as corticosteroids, azathioprine, anti-inflammatory medications, or other agents that can either directly or indirectly influence the risk of thrombosis and bleeding and which may interact with one of the these newer agents, increasing or decreasing drug levels $[5,12]$. This is particularly important as there is no reliable method of monitoring DOAC therapy. Third, patients with APS are different than those enrolled in the clinical trials that have demonstrated the efficacy of the DOACs; they are generally younger and have both chronic and acute comorbid conditions which may influence the risk of both first and recurrent thrombosis (e.g., concomitant inflammatory disorders, immobilization, renal insufficiency, and the need for surgical procedures). Taken in sum, these observations suggest that before DOACs are widely adopted in patients with APS, we require good quality evidence for their efficacy and safety.

Given the uncertainty regarding the safety and efficacy of DOACs in patients with APS, we initiated a research program dedicated to this question. A large definitive study powered to detect clinically important outcomes would be required to address this question; however, prior to mounting such a large and expensive study, we carried out a pilot feasibility study. Our rationale for a pilot study was as follows: APS is a rare disease and eligible consenting patients may be difficult to identify and enroll despite the participation of 6 clinical centers; patients with APS may have a strong preference for continuing to receive warfarin given the evidence of benefit from previous studies; and adherence, if suboptimal, may pose safety considerations given the potential increased risk of thrombosis in the setting of short-term interruptions in rivaroxaban administration. A pilot study would thus allow for a more accurate estimate of enrollment capacity and consent rates, provide information regarding expected adherence, and allow for an estimate of rates of thrombosis and bleeding to inform a larger definitive study.

In this pilot feasibility study, we explored the following feasibility outcomes: (a) we examined our ability to identify 150 eligible APS patients; (b) we measured our ability to obtain consent from 135 of these patients; and (c) we tested our hypothesis that we could obtain $95 \%$ adherence with daily rivaroxaban administration.

\section{Material and methods}

Adult ( $\geq 18$ years) patients fulfilling criteria for APS [1] who had prior VTE and who did not have a contraindication to use of rivaroxaban were recruited to participate at 6 clinical sites in Canada (St. Joseph's Healthcare Hamilton, Hamilton, ON; Hamilton Health Sciences, Hamilton, ON; Jewish General Hospital, Montreal, QC; QEII Health Sciences Centre, Halifax, NS; The Ottawa Hospital, Ottawa, ON; and University of Alberta Hospital, Edmonton, $\mathrm{AB}$ ) over a 2-year period (October 2014-September 2016). Exclusion criteria included previous recurrent thrombosis while taking warfarin, need for therapy with either rivaroxaban $2.5 \mathrm{mg}$ or $5 \mathrm{mg}$ BID or both aspirin and clopidogrel for acute coronary syndrome (ACS), pregnancy, chronic renal insufficiency with glomerular filtration rate $<30$, geographic inaccessibility, inability to provide informed consent, or situation where, in the opinion of the treating physician, rivaroxaban is to be avoided (e.g., excessive bleeding risk, hypersensitivity), or another anticoagulant is preferred (e.g., mechanical heart valve, active malignancy). In addition, as rivaroxaban $20 \mathrm{mg}$ daily is not approved in Canada for secondary prevention of thrombosis in patients with ACS or stroke, patients with isolated arterial thromboembolism as the sole manifestation of APS were also excluded.

All successfully recruited patients were followed for a minimum of 1 year (follow-up period completed September 2017). If patients were eligible to participate but declined consent, the reason for declining participation was collected, if possible. All consenting participants were transitioned from their current anticoagulant to rivaroxaban $20 \mathrm{mg}$ orally daily. Patients were reassessed clinically every 3 months by telephone or in-person visits to collect data on thrombotic events, bleeding events, or other adverse events. In-person visits occurred at minimum every 6 months, when pill counts were completed to determine adherence. Informed consent was obtained from all participants, and research ethics board approval for the study was obtained from each clinical site 
(Hamilton Integrated Research Ethics Board identifier for central site: \#14-366). Independent adjudicators assessed thromboembolic and bleeding events. An independent safety monitor reviewed all adverse events. Two levels of safety monitoring were built in-informal consecutive patient monitoring and formal analyses after 50 and 100 recruited patients (if reached) achieved 6 months of followup. Given that the estimated frequencies of both thrombosis and bleeding from previous warfarin studies were 3$6 \%$, the upper limit of $6 \%$ was chosen as the safety limit for this study. The frequencies of adjudicated thrombotic and bleeding events were separately calculated with a 97.5\% confidence interval (using significance of 0.05 for the set of tests and Bonferroni correction applied for two independent tests) at the two monitoring levels during the study. Using this method, 10 bleeding or thrombotic events would have to have been recorded for the first 50 patients, or 14 for the first 100 patients, to trigger a safety concern. The study was registered with clinicaltrials.gov, identifier NCT02116036. The pilot trial protocol can be accessed from the corresponding author upon request.

\section{Outcome measures}

\section{Primary outcomes (feasibility objectives)}

- Enrolment-We defined success as the ability to identify 150 eligible patients over the study period.

- Consent-We defined success as a consent rate of 90\% (thus, with identification of 150 patients, at least 135 would have provided consent).

- Compliance-We defined success as missing fewer than $5 \%$ of days with pill administrations.

\section{Secondary outcome measures}

- Bleeding-rates of major and minor bleeding were collected.

- Major bleeding was defined using International Society of Thrombosis and Haemostasis criteria [13]

- Minor bleeding was defined as all bleeding that did not meet criteria for major bleeding

- Thrombosis-rates of VTE and arterial thrombosis were collected. Outcome definitions for VTE, myocardial infarction, ischemic stroke, and cardiovascular death were adapted from the COMPASS study [14].

Data was recorded through Research Electronic Data Capture (REDCap) [15]. Data analysis was performed by comparing recruitment rates to the pre-specified feasibility endpoints and by calculating rates of thrombosis and bleeding events. The sample size for the study, i.e., 135 patients followed for a minimum of 1 year, was chosen to allow us to obtain estimates of bleeding and thrombosis rates in addition to the assessments of recruitment rates and adherence. The rationale was that if recruitment had occurred as planned, the average follow-up of enrolled patients would be about 16 months, providing about 180 patient-years of exposure. This exposure was expected to be sufficient to accrue between 4 and 8 adjudicated bleeding and thrombotic events, allowing us to calculate estimates of bleeding and thrombosis rates with reasonable $95 \%$ confidence intervals.

\section{Results}

\section{Included patients}

Ninety-six patients meeting inclusion/exclusion requirements were identified over the 2-year period from October 2014 to September 2016, and 14 patients declined participation (85\% consent rate). Fourteen of 96 eligible patients declined consent, with the reasons stated being desire to remain on warfarin therapy (7/14), logistical (e.g., difficulty getting to study center for study visits, 2/ 14), or no clear stated reason (e.g., "not interested," 5/ 14). The resulting 82 patients were followed for a mean of 19.0 months (median 21 months), ending September 2017, representing 129.8 patient-years of data. Baseline characteristics are described in Table 1; 10 patients (8.2\%) had SLE. Forty-seven patients (57.3\%) were single-positive for antiphospholipid antibodies (APLA) (i.e., had one of LA, aCL, or a $\beta 2 \mathrm{GP} 1$ positive), 11 patients (13.4\%) were double-positive (i.e., had two of $\mathrm{LA}, \mathrm{aCL}$, and/or a $32 \mathrm{GP} 1$ positive), and no patient was triple-positive (i.e., were $\mathrm{LA}, \mathrm{aCL}$, and a $\beta 2 \mathrm{GP} 1$ positive). Notably, 24 of the patients in the study were found at analysis not to have fulfilled the Sapporo criteria for persistent APLA positivity, having either had only one isolated positive result, or aCL and/or a $32 \mathrm{GP} 1$ at low-titer

Table 1 Baseline demographics of included patients

\begin{tabular}{ll}
\hline Demographic & $N(\%)$ or result \\
\hline Female sex & $39(47.6 \%)$ \\
Average age at study entry & 53.4 years \\
Previous arterial events & $5(6 \%)$ \\
$\geq 2$ previous thromboses & $24(29.2 \%)$ \\
On warfarin at study entry & $41(50 \%)$ \\
On rivaroxaban at study entry & $38(46.3 \%)$ \\
LA positive & $46(56 \%)$ \\
aCL positive & $30(36.6 \%)$ \\
aß2GP1 positive & $4(4.9 \%)$ \\
Double positivity & $11(13.4 \%)$ \\
Triple positivity & $0(0 \%)$ \\
SLE & $10(8.2 \%)$ \\
\hline
\end{tabular}

$a C L$ anticardiolipin, $a \beta 2 G P 1$ anti- $\beta 2$ glycoprotein-1, $L A$ lupus anticoagulant, SLE systemic lupus erythematosus 
only, though all patients were considered to have APS by the treating clinician.

\section{Medication adherence}

Fifty-five patients (67\%) had pill counts performed on at least one occasion. One of these patients had unusable data due to the use of inpatient supply during hospitalization; thus, data from 54 patients were used for adherence determination. Forty-four of 54 patients $(81 \%)$ achieved $>95 \%$ adherence. Average adherence was $95.0 \%$.

\section{Clinical outcomes}

Over the follow-up period, there were 4 recurrent thromboembolic events $(2$ arterial cerebrovascular events, 1 deep vein thrombosis, and 1 pulmonary embolus) (3.0 events/100 patient-years) in patients taking rivaroxaban. Characteristics of patients experiencing a recurrent event are described in Table 2. There were two thrombotic episodes in patients who were no longer taking rivaroxaban at the time of the event: One patient had VTE while receiving low molecular weight heparin after development of hepatitis secondary to disseminated histoplasmosis necessitating discontinuation of rivaroxaban, and a second patient had ischemic stroke while on warfarin after having rivaroxaban discontinued due to headache and hypertension. None of the patients who experienced recurrent thromboses had SLE.

There were 23 episodes of minor bleeding, and no episodes of major bleeding. In 3 of these episodes, specific medical intervention was required to control bleeding. Eight of these patients had rivaroxaban held temporarily, and one patient discontinued study drug.

Thirty-two patients had a total of 45 reported adverse events during the study, though there were no serious adverse events (Table 3). Ten of these adverse events were drug-related or possibly drug-related. Nine events required permanent discontinuation of study medication. A further 6 patients withdrew consent partway through the study or were lost to follow-up.
Full study data can be accessed upon request from the corresponding author. CONSORT Extension to Pilot and Feasibility Trials checklist is attached as Additional file 1.

\section{Discussion}

The study did not meet its feasibility endpoints, with only 96 of a predicted 150 patients identified and 82 of a predicted 135 patients consenting to participate in the study (representing an $85 \%$ consent rate, less than the $90 \%$ predicted a priori), despite the participation of 6 clinical centers in Canada with expertise in APS. The inclusion only of patients with a history of venous thromboembolic manifestations led to exclusion of patients with isolated arterial events (in Canada, rivaroxaban $20 \mathrm{mg}$ daily is off-label both for cerebrovascular and cardiovascular events; thus such patients were ineligible for the study), which may have affected enrolment appreciably given that in some cohorts arterial events are more common than venous events in patients with APS [16]. Future studies could consider recruitment of patients with a history of isolated arterial events which may improve feasibility of this study design. The two most commonly cited reasons for patients declining to participate in the study were logistical issues, including concern regarding travel to the study center for visits, and patient concern regarding comparative efficacy of the study medication versus warfarin leading them to opt to remain on warfarin. It is difficult to modify the study protocol appreciably to address either of these concerns; from a logistical point of view, the study required in-person visits only every 6 months-a longer time interval such as annual visits could be considered, however would be likely to further affect the adherence measurements, where missing data were already an important concern.

The study technically met its adherence data endpoint where average adherence using the available pill count data was $95.0 \%$; however, there is a risk of bias on this estimate given the amount of missing data for this

Table $\mathbf{2}$ Characteristics of patients with recurrent thrombotic event

\begin{tabular}{lllll}
\hline Characteristic & Patient 1 & Patient 2 & Patient 3 & Patient 4 \\
\hline Location of thrombus & MCA stroke & PE & MCA stroke & DVT \\
Age & 33 years & 19 years & 64 years & 54 years \\
Sex & F & M & M & F L, titer 23 \\
aPL profile & LA & LA & 2 & LA \\
Number of previous thromboses at baseline & 4 & 1 & $\mathrm{~N}$ & $\mathrm{~N}$ \\
Prev arterial thrombosis? & Y & $\mathrm{N}$ & $\mathrm{N}$ \\
SLE? & $\mathrm{N}$ & $\mathrm{N}$ & $\mathrm{N}$ \\
$>95 \%$ adherence? & Y & Y & No bottles returned
\end{tabular}

$a C L$ anticardiolipin antibody, $a P L$ antiphospholipid antibody, $D V T$ deep venous thrombosis, MCA middle cerebral artery, $L A$ lupus anticoagulant, $P E$ pulmonary embolism, SLE systemic lupus erythematosus 
Table 3 Adverse events

\begin{tabular}{|c|c|c|}
\hline Adverse Event(s) & Rivaroxaban-related? $(\mathrm{n}=)$ & Rivaroxaban discontinued? $(\mathrm{n}=)$ \\
\hline $\begin{array}{l}\text { Infectious (Influenza, respiratory tract, fever NYD, cellulitis, } \\
\text { pneumosepsis, disseminated histoplasmosis, viral infection NOS) n=9 }\end{array}$ & $\mathrm{n}=0$ & $\mathrm{n}=1$ \\
\hline $\begin{array}{l}\text { Gastrointestinal (Nausea, melena, colonoscopy, } 20 \text { lb weight gain, } \\
\text { epigastric pain) } n=8\end{array}$ & $n=2$ & $n=1$ \\
\hline Headache $n=5$ & $n=4$ & $n=2$ \\
\hline Dermatological (Rash, finger nodules, leg ulcer) $n=4$ & $n=1$ & $\mathrm{n}=2$ \\
\hline Neurological (Peripheral neuropathy/CNS impairment NYD, Bell's palsy) $n=3$ & $\mathrm{n}=0$ & $\mathrm{n}=0$ \\
\hline SLE flare (including class IV lupus nephritis) $n=2$ & $\mathrm{n}=0$ & $n=1$ \\
\hline $\begin{array}{l}\text { Other (Chest pain, broken tooth, right hip arthroplasty, back pain, } \\
\text { depression, cough, post-thrombotic syndrome, felt unwell, joint pain, } \\
\text { prostate cancer, hypertensive urgency, SOB NYD) } n=10\end{array}$ & $\mathrm{n}=0$ & $n=2$ \\
\hline Death (Pneumosepsis) $n=1$ & $\mathrm{n}=0$ & N/A \\
\hline
\end{tabular}

outcome. No patient had a complete pill count performed for the duration of the study, generally due to patients only returning a portion (usually the last 3 months' worth) of bottles at each 6-month in-person visit, and a full one-third of the study subjects never returned any bottles at all. However, even with higher rate of return of study bottles, pill counts have been shown to be an imperfect measure of adherence $[17,18]$. More robust methods for such determination could be considered, such as laboratory monitoring of anti-Xa activity or electronic pill bottles that record timing of use, though these typically increase the complexity and expense of a study and future studies must balance these competing interests [19]. Ensuring adherence to rivaroxaban in this population is of importance, as its short half-life poses a risk of under-anticoagulation compared to warfarin.

The study does provide efficacy and safety data on 129.8 patient-years of rivaroxaban in APS, making it one of the larger cohorts reported to date. Demographics of patients in our cohort were relatively similar to those of other cohorts of APS patients: approximately 50\% were female, with average age in the early 50's. There were no unexpected adverse events reported for rivaroxaban, and no episodes of major bleeding in this cohort. While there was no comparator group, the rate of recurrent thromboembolic events falls close to the rates seen in the older randomized controlled trials (RCTs) of warfarin use in APS to date (Table 4) $[4,20]$. Notably, however, the two most recent RCTs of warfarin in APS have not demonstrated any thromboembolic events in the warfarin groups, which is somewhat surprising given the high-risk features of most of the enrolled patients [21, 22]. It must be noted that the patients enrolled in our study were at relatively low risk of recurrent thrombosis compared to many in the APS population-no patients had had previous failure of warfarin therapy, no patients were "triple positive," and $20 \%$ of the study sample had only low-titer or one-time positive aCL or aß2GP1. Of the 4 patients who had recurrent thromboembolism, 3 patients were LA positive, which may reflect the slightly higher risk of thrombosis in patients who have isolated LA positivity compared to those with an aCL or a $\beta 2 \mathrm{GP} 1$ antibody [23]. The results from our study are in contrast with the recent study by Pengo et al., where a significant imbalance in the composite endpoint of thromboembolic events, major bleeding, and vascular death was seen between patients receiving rivaroxaban compared to warfarin for "triple positive" patients [11 patients in the rivaroxaban group versus 2 patients in the warfarin group (hazard ratio 6.7, 95\% CI 1.5-30.5; $p=0.01$ )] leading to premature study discontinuation [22]. There were 7 arterial events in the rivaroxaban group compared to 0 in the warfarin group, and 4 episodes of major bleeding

Table 4 Comparison of standardized rates of thrombosis in our study versus previous randomized controlled trials of warfarin in antiphospholipid syndrome

\begin{tabular}{|c|c|c|c|c|c|}
\hline & Our study & Crowther, 2003 & Finazzi, 2005 & Cohen, 2016 & Pengo, 2018 \\
\hline No. of patients (No. with arterial events at baseline) & $82(5)$ & $114(27)$ & $109(44)$ & $116(0)$ & Warfarin arm: 61 (14) \\
\hline Length of follow-up & 19 months (mean) & 2.7 years (mean) & 3.6 years (median) & 6 months & 569 days (mean) \\
\hline No. of recurrent VTE events (\%) & $2(2.4)$ & $5(4.4)$ & $3(2.3)$ & $0(0)$ & $0(0)$ \\
\hline No. of recurrent arterial events (\%) & $2(2.4)$ & $3(2.6)$ & $7(6.4)$ & $0(0)$ & $0(0)$ \\
\hline Recurrent thrombotic events/100 patient-years & 3.1 & 2.6 & 2.5 & 0 & 0 \\
\hline
\end{tabular}


compared to 2 in the warfarin group. Overall, this study does not reveal a high risk of recurrent thromboembolic disease in patients on rivaroxaban; however, it must be noted that this is likely to be a relatively low-risk cohort of APS patients, and this study did not have a control group.

Limitations of the study include (1) inclusion only of the subset of APS patients with venous thromboembolism, excluding the subset with isolated arterial events, which may have affected enrolment; (2) the suboptimal rate of return of study bottles for pill count; and (3) the differing antibody profiles of our patients compared to those in other studies of APS patients, with a high number of patients not fulfilling Sapporo criteria for APS.

In conclusion, this study shows that adequately powered comparative trials using clinical outcomes in APS are unlikely to be successful due to inability to recruit sufficient numbers of study subjects, even when recruiting through multiple centers with specific APS expertise. This study does not reveal a higher than expected risk of recurrent thromboembolic disease compared to historical cohorts; however, it is noteworthy that this is an uncontrolled study in relatively low-risk APS patients.

\section{Supplementary information}

Supplementary information accompanies this paper at https://doi.org/10. 1186/s40814-020-00594-1.

Additional file 1. CONSORT 2010 checklist of information to include when reporting a pilot or feasibility trial

\section{Abbreviations}

aß2GP1: Anti-beta-2 glycoprotein-1 antibody; aCL: Anticardiolipin antibody; ACS: Acute coronary syndrome; APLA: Antiphospholipid antibodies; APS: Antiphospholipid syndrome; DVT: Deep venous thrombosis; DOAC: Direct oral anticoagulant; INR: International normalized ratio; LA: Lupus anticoagulant; MCA: Middle cerebral artery; PE: Pulmonary embolism; RCT: Randomized controlled trial; SLE: Systemic lupus erythematosus; VTE: Venous thromboembolism

\section{Acknowledgements}

Not applicable

\section{Authors' contributions}

$\mathrm{KL}$ wrote the study protocol, performed research, analyzed data, and wrote the study manuscript. MB performed the research and reviewed, edited, and approved the manuscript. MC performed the research and reviewed, edited, and approved the manuscript. SK performed the research and reviewed, edited, and approved the manuscript. S. Schulman performed the research and reviewed, edited, and approved the manuscript. S. Shivakumar performed the research and reviewed, edited, and approved the manuscript. CW performed the research and reviewed, edited, and approved the manuscript. MAC obtained the funding, wrote the study protocol, performed the research, and reviewed, edited, and approved the manuscript. The manuscript has been read and approved for submission by all qualified authors.

\section{Funding}

The source of financial support for this study was through the Heart and Stroke Foundation of Canada (grant number G-13-0002011). The study also received support (including in-kind support of study rivaroxaban) from Bayer. The sponsors did not participate in the study design, collection/analysis/ interpretation of data, in writing of the report, nor in the decision to submit the article for publication; all activities and decisions were carried out by the study authors.

\section{Availability of data and materials}

The datasets used and/or analyzed during the current study are available from the corresponding author on reasonable request.

Ethics approval and consent to participate

Research ethics board approval for the study was obtained from each clinical site (Hamilton Integrated Research Ethics Board identifier for central site: \#14366). Written consent was obtained from each participant.

\section{Consent for publication}

Not applicable

\section{Competing interests}

This study received support from Bayer. MB has received advisory board fees and research support from Bayer. MC has received research funding from BMS and Leo Pharma and honoraria from Pfizer, Bayer, Leo Pharma, and Sanofi. SK has received advisory board fees from BMS, Pfizer, Sanofi, and Aspen. S. Shivakumar has received honoraria from Bayer and Pfizer. CW has received advisory board fees for Leo pharma and Pfizer. KL, MAC, S. Schulman declared no additional COI.

\section{Author details}

${ }^{1}$ Department of Medicine, McMaster University, 1280 Main St. W, Hamilton, ON L8S 4L8, Canada. ${ }^{2}$ Department of Medicine, McGill University, 845 Sherbrooke St. W., Montreal, QC H3A 2T5, Canada. ${ }^{3}$ Department of Medicine, University of Ottawa, 75 Laurier Ave E, Ottawa, ON K1N 6N5, Canada. ${ }^{4}$ Department of Medicine, Dalhousie University, 6299 South St, Halifax, NS B3H 4R2, Canada. ${ }^{5}$ Department of Medicine, University of Alberta, 116 St \& 85 Ave, Edmonton, AB T6G 2R3, Canada.

Received: 5 April 2019 Accepted: 3 April 2020

Published online: 25 April 2020

\section{References}

1. Miyakis S, Lockshin MD, Atsumi T, et al. International consensus statement on an update of the classification criteria for definite antiphospholipid syndrome (APS). J Thromb Haemost. 2006:4:295-306.

2. Hamsten A, Norberg R, Björkholm M, et al. Antibodies to cardiolipin in young survivors of myocardial infarction: an association with recurrent cardiovascular events. Lancet. 1986;1:113-6.

3. Bertero MT, Bazzan M, Carignola R, et al. Antiphospholipid syndrome in northwest Italy (APS Piedmont Cohort): demographic features, risk factors, clinical and laboratory profile. Lupus. 2010:21:806-9.

4. Crowther MA, Ginsberg JS, Julian J, et al. A comparison of two intensities of warfarin for the prevention of recurrent thrombosis in patients with the antiphospholipid antibody syndrome. N Engl J Med. 2003;349:1133-8.

5. Ageno W, Gallus AS, Wittkowsky A, et al. Oral anticoagulant therapy: antithrombotic therapy and prevention of thrombosis, 9th ed: American College of Chest Physicians Evidence-Based Clinical Practice Guidelines. Chest. 2012;141:e44S-88S.

6. Ahmad Y, Lip GYH. Stroke prevention in atrial fibrillation: where are we now? Clin Med Insights Cardiol. 2012;6:65-78.

7. Bauersachs R, Berkowitz SD, Brenner B, et al. Oral rivaroxaban for symptomatic venous thromboembolism. N Engl J Med. 2010;363:2499-510.

8. Buller HR, Prins MH, Lensin AWA, et al. Oral rivaroxaban for the treatment of symptomatic pulmonary embolism. N Engl J Med. 2012;366:1287-97.

9. Schulman S, Kearon C, Kakkar AK, et al. Dabigatran versus warfarin in the treatment of acute venous thromboembolism. N Engl J Med. 2009;361: 2342-52.

10. Rosove MH, Brewer PM. Antiphospholipid thrombosis: clinical course after the first thrombotic event in 70 patients. Ann Intern Med. 1992:117:303-8.

11. Khamashta MA, Cuadrado MJ, Mujic F, et al. The management of thrombosis in the antiphospholipid-antibody syndrome. N Engl J Med. 1995;332:993-7.

12. Holbrook AM, Pereira JA, Labiris R, et al. Systematic overview of warfarin and its drug and food interactions. Arch Intern Med. 2005;165:1095-106. 
13. Schulman S, Kearon C, Subcommittee on Control of Anticoagulation of the Scientific and Standardization Committee of the International Society on Thrombosis and Haemostasis. Definition of major bleeding in clinical investigations of antihemostatic medicinal products in non-surgical patients. J Thromb Haemost. 2005;3:692-4.

14. Connolly SJ, Eikelboom JW, Bosch J, et al. Rivaroxaban with or without aspirin in patients with stable coronary artery disease: an international, randomised, double-blind, placebo-controlled trial. Lancet. 2018;391:205-18.

15. Harris PA, Taylor R, Thielke $R$, et al. Research electronic data capture (REDCap)--a metadata-driven methodology and workflow process for providing translational research informatics support. J Biomed Inform. 2009; 42:377-81.

16. Cervera R, Serrano R, Pons-Estel GJ, et al. Morbidity and mortality in the antiphospholipid syndrome during a 10-year period: a multicentre prospective study of 1000 patients. Ann Rheum Dis. 2015;74:1011-8.

17. Rudd P, Byyny RL, Zachary V, et al. Pill count measures of compliance in a drug trial: variability and suitability. Am J Hypertens. 1988:1:309-12.

18. Rudd P, Byyny RL, Zachary V, et al. The natural history of medication compliance in a drug trial: limitations of pill counts. Clin Pharmacol Ther. 1989:46:169-76.

19. Jayaraman S, Rieder MJ, Matsui DM. Compliance assessment in drug trials: has there been improvement in two decades? Can J Clin Pharmacol. 2005; 12:e251-3.

20. Finazzi $G$, Marchioli R, Brancaccio V, et al. A randomized clinical trial of highintensity warfarin vs. conventional antithrombotic therapy for the prevention of recurrent thrombosis in patients with the antiphospholipid syndrome (WAPS). J Thromb Haemost. 2005;3:848-53.

21. Cohen $H$, Hunt BJ, Efthymiou M, et al. Rivaroxaban versus warfarin to treat patients with thrombotic antiphospholipid syndrome, with or without systemic lupus erythematosus (RAPS): a randomised, controlled, open-label, phase 2/3, non-inferiority trial. Lancet Haematol. 2016;3:e426-36.

22. Pengo V, Denas $G$, Zoppellaro $G$, et al. Rivaroxaban vs warfarin in high-risk patients with antiphospholipid syndrome. Blood. 2018;132:1365-71.

23. Galli $M$, Luciani $D$, Bertolini $G$, et al. Lupus anticoagulants are stronger risk factors for thrombosis than anticardiolipin antibodies in the antiphospholipid syndrome: a systematic review of the literature. Blood. 2003;101:1827-32

\section{Publisher's Note}

Springer Nature remains neutral with regard to jurisdictional claims in published maps and institutional affiliations.

Ready to submit your research? Choose BMC and benefit from:

- fast, convenient online submission

- thorough peer review by experienced researchers in your field

- rapid publication on acceptance

- support for research data, including large and complex data types

- gold Open Access which fosters wider collaboration and increased citations

- maximum visibility for your research: over $100 \mathrm{M}$ website views per year

At $\mathrm{BMC}$, research is always in progress.

Learn more biomedcentral.com/submissions 